\title{
Fuzzy Inference Systems Implemented on Neural Architectures for Motor Fault Detection and Diagnosis
}

\author{
Sinan Altug, Mo-Yuen Chow, Senior Member, IEEE, and H. Joel Trussell, Fellow, IEEE
}

\begin{abstract}
Motor fault detection and diagnosis involves processing a large amount of information of the motor system. With the combined synergy of fuzzy logic and neural networks, a better understanding of the heuristics underlying the motor fault detection/diagnosis process and successful fault detection/diagnosis schemes can be achieved. This paper presents two neural fuzzy (NN/FZ) inference systems, namely, Fuzzy Adaptive Learning Control/Decision Network (FALCON) and Adaptive Network Based Fuzzy Inference System (ANFIS), with applications to induction motor fault detection/diagnosis problems. The general specifications of the NN/FZ systems are discussed. In addition, the fault detection/diagnosis structures are analyzed and compared with regard to their learning algorithms, initial knowledge requirements, extracted knowledge types, domain partitioning, rule structuring and modifications. Simulated experimental results are presented in terms of motor fault detection accuracy and knowledge extraction feasibility. Results suggest new and promising research areas for using NN/FZ inference systems for incipient fault detection and diagnosis in induction motors.
\end{abstract}

Index Terms - Fuzzy inference systems, induction motors, knowledge extraction, motor fault diagnosis, neural/fuzzy systems.

\section{INTRODUCTION}

\section{A. Importance of Motor Fault Detection and Diagnosis for Industrial Applications}

I NDUCTION motors are the workhorses of industry because of their roughness and versatility. Different articles have discussed the key issues for successful motor operation: a quality motor, understanding of the application, choice of the proper type of motor for the application, and proper maintenance of the motor. However, the use of induction motors in today's industry is extensive, and the motors can be exposed to different hostile environments, misoperation, manufacturing defects, etc. Different internal motor faults (e.g., short circuit of motor leads, interturn short circuits, ground faults, worn out/broken bearings, broken rotor bars) along with external motor faults (e.g., phase failure, asymmetry of mains supply, mechanical overload, blocked rotor, underload) are expected to happen sooner or later. Furthermore, the wide variety of environments and conditions that the motors are exposed to

Manuscript received April 4, 1997; revised May 7, 1999. Abstract published on the Internet August 20, 1999. This work was supported by the National Science Foundation under Grant ECS-9521609 and Grant ECS-9610509.

The authors are with the Department of Electrical and Computer Engineering, North Carolina State University, Raleigh, NC 27695-7911 USA.

Publisher Item Identifier S 0278-0046(99)08472-5. can age the motor and make it subject to incipient faults [1]-[3]. These types of faults usually refer to the gradual deterioration in the motor that can lead to motor failure if left undetected. Motor problems can cause crises that are expensive and are quite annoying, in particular, if the problem could have been prevented. Actually, many motor faults could be avoided if the application, environment, and the cause-effect of motor faults were understood [4]. Therefore, reliability demands for electric motors are constantly increasing due to some of the important motor applications, and the advancement in technologies.

\section{B. Importance of System Monitoring and Fault Detection}

Many engineers and researchers have focused on incipient fault detection and preventive maintenance, which aim at preventing motor faults from happening [5]-[9]. Usually, devices such as fuses, overload relays, and circuit breakers protect induction motors. Research has focused on different motor failure mechanisms, causes of stator and rotor failures, analyses of these failures, methodologies to determine whether a motor is suitable for extended service, test methods, the test equipment needed, application and limitations of these test procedures, data gathering, specific benefits, and costs [10], [11]. In addition to developing motor protection schemes in reaction to faults due to misoperation, disturbances, sudden failure, etc., motor incipient fault detection problems have also been attracting significant attention and interest. Online monitoring of induction machines in critical applications has been increasingly necessary to improve their reliability and to minimize catastrophic failures. Microprocessor-based monitoring systems are of particular interest because they can be used for regular analysis of machine variables and to predict possible fault conditions, so that preventive maintenance can be organized in a cost-effective manner. Different researchers have addressed the importance and economic benefits of online motor monitoring and fault detection approaches [1], [11]. General methods of cost-benefit analysis have been applied to investigate the financial viability of such systems. Methods for the evaluation of the improvement of machine reliability by monitoring of systems have also been discussed [12].

Different invasive and noninvasive approaches for motor incipient fault detection/diagnosis have been reported [2], [5]-[8]. Many of the motor incipient fault detection/diagnosis schemes can be applied noninvasively on-line without the need 
of expensive monitoring equipment by using a microprocessor. With proper monitoring and fault detection/diagnosis schemes, the incipient faults can be detected in their early stages; thus, maintenance and downtime expenses can be reduced, and reliability can be improved. System identification and parameter estimation have previously been proposed for fault detection/diagnosis in motors [5]-[8], [13], [14]. As opposed to conventional techniques, where expensive equipment or accurate mathematical models are required, fuzzy logic and neural network (NN) technologies can be used to provide inexpensive but effective fault detection mechanism alternatives [15].

\section{Fuzzy Logic and NN Technologies for Fault Detection}

Fuzzy-rule-base modeling is to identify the structures and the parameters of a fuzzy if-then rule base so that a desired input/output mapping is achieved. Recently, using adaptive networks to fine tune membership functions of a fuzzy rule base has received more attention [16]. Many methods have been proposed for implementing and optimizing fuzzy reasoning via NN structures [15]-[21]. Parameters in fuzzy systems have clear physical meanings so that rule-based and linguistic information can be incorporated into adaptive fuzzy systems systematically. On the other hand, there exist powerful algorithms to train various NN models to adapt difficult input-output mappings. The idea behind the fusion of these two technologies is to use the learning ability of NN's to implement and automate the fuzzy systems, which utilize the high-level human-like reasoning capability [22].

Different NN structures have proven to be successful in solving the motor fault detection/diagnosis problem [1], [15], [23]-[25]. Following the techniques developed for general process fault detection/diagnosis [5]-[8], [26], and system identification via NN's [27], [28], the studies on the use of NN's for fault detection have been structured around several concepts. One of these concepts is to estimate system output, given a number of previous input and output values [24]. Another approach is to train the $\mathrm{NN}$ for on-line or off-line estimation of certain system parameters. The $\mathrm{NN}$ is trained to estimate system parameters under different fault conditions using appropriate inputs and outputs (and/or certain observed variables) of the system, in a supervised learning environment. References [1], [9], [23]-[25], and [29] present similar approaches to design feedforward artificial NN's to perform motor fault detection/diagnosis. Human expert approaches and the use of fuzzy logic to optimize such NN structures were presented in [30].

The artificial NN fault detection/diagnosis method, by itself, cannot provide heuristic knowledge of the motor or the fault detection process because of its blackbox approach [2]. On the other hand, fuzzy logic is a tool that can easily implement and utilize heuristic reasoning, but it is, in general, difficult to provide exact solutions. Fuzzy sets have been used for fault diagnosis [31]-[33]. However, most of these schemes are "static," i.e., a general fuzzy inference system is formed and is not allowed to change throughout the experiments. The faults are classified using this static inference engine, rather than adapting to different operating conditions.
With the combined synergy of fuzzy logic and NN's, a better understanding of the detection/diagnosis process of the system can be achieved and, also, the fault detector can be adapted to provide more accurate solutions under different operation conditions. In spite of the fact that there has been extensive research on artificial NN's for motor fault detection/diagnosis, the use of a hybrid neural/fuzzy system is a fairly new concept in the motor fault detection area. Most of the studies in this area are application oriented. A detailed methodology is presented in [15], where a hybrid neural/fuzzy fault detector is used to solve the motor fault detection problem. In these applications, the neural/fuzzy fault detector is used to monitor the condition of the motor bearing and the insulation. The fault detector not only provides accurate fault detector performance, but also the heuristic reasoning behind the fault detection process and the actual motor fault conditions.

In [34], an adaptive neural fuzzy (NN/FZ) system was used to classify faults in a power system and to bring heuristic explanation to the process. Other approaches using an NN/FZ system include [35], where an NN/FZ model is used to detect faults in nonlinear dynamic systems. In other studies, an adaptive threshold test based upon fuzzy modeling of the process is employed [36]. Another scheme is given in [37], where the dynamical update of three parameters of the $\mathrm{NN}$, the training rate, momentum, and the activation function slope, is performed by a fuzzy structure. Another technique is to add a fuzzification layer to a conventional feedforward $\mathrm{NN}$ to track faults [38].

This paper focuses on the applications of two popular NN/FZ structures to solve the induction motor fault detection/diagnosis problem: the Fuzzy Adaptive Learning Control/Decision Network (FALCON)-Based Fault Detector (FFD), and the Adaptive-Network-Based Fuzzy Inference System (ANFIS)-Based Fault Detector (AFD). The complete methodology for employing these structures for motor fault detection is provided. In Section II, the issues related to the construction of fault detectors for induction motors are discussed. Section III briefly describes the architectures and training procedures of the NN/FZ systems. Section IV analyzes and compares the FFD and AFD motor fault detector structures with respect to their learning algorithms, initial knowledge requirements, extracted knowledge types, domain partitioning, and rule structuring and revising. Section V gives the comparative evaluation of simulated experimental results in terms of motor fault detection accuracy and knowledge extraction capability. Section VI is the discussion and conclusions.

\section{Description of the Motor FAUlt Detection Problem}

\section{A. Induction Motor System}

In a three-phase induction motor framework, stator currents $i$ and rotor angular velocity $\omega$ are measured under different motor friction $f$ and load torque $\tau$. The magnitude of motor friction and load torque affect motor operations, which, in turn, affect speed and current measurements. However, the magnitude of the motor friction cannot be measured directly. 


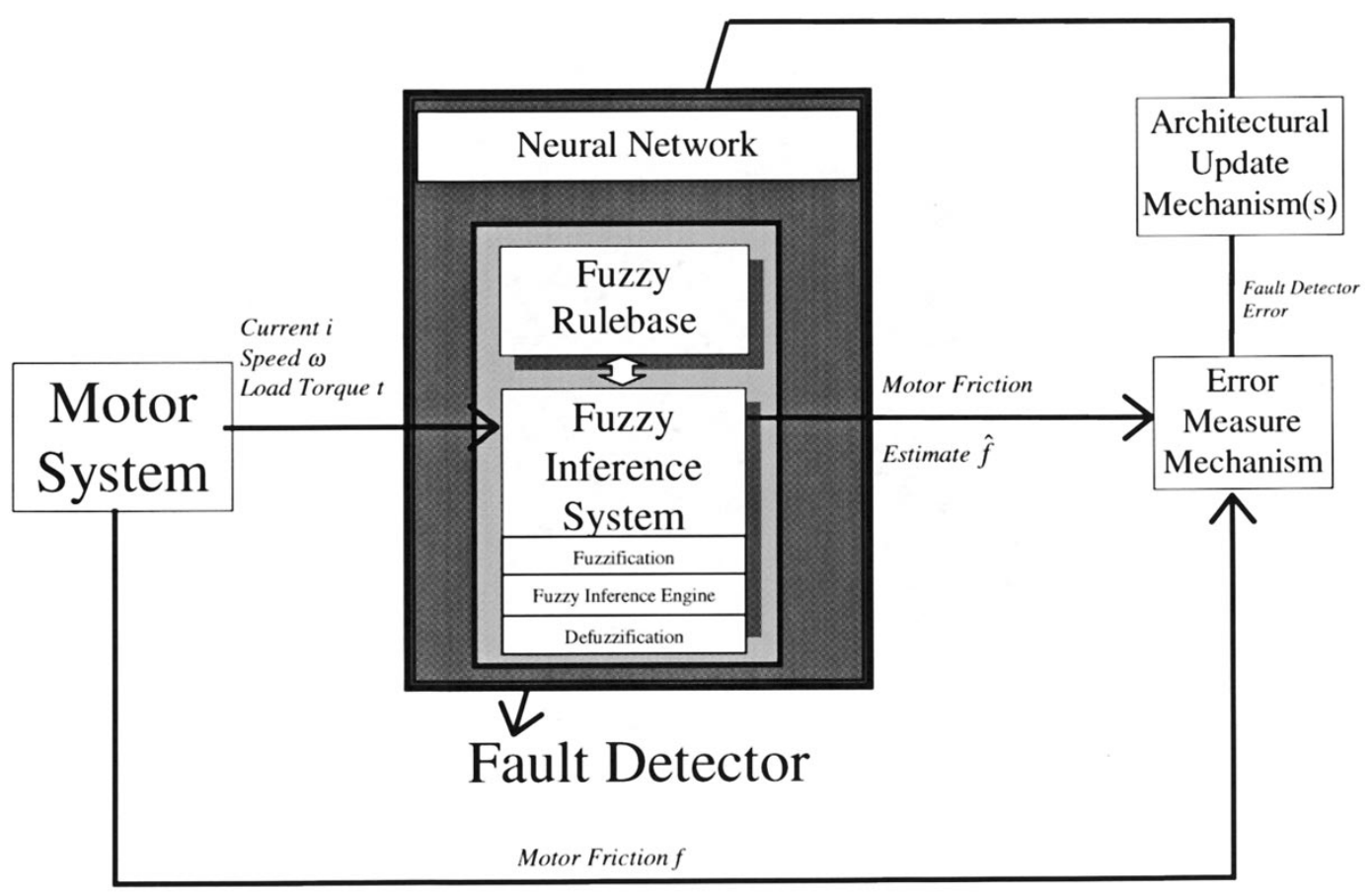

Fig. 1. Conceptual diagram of the fault detector structure.

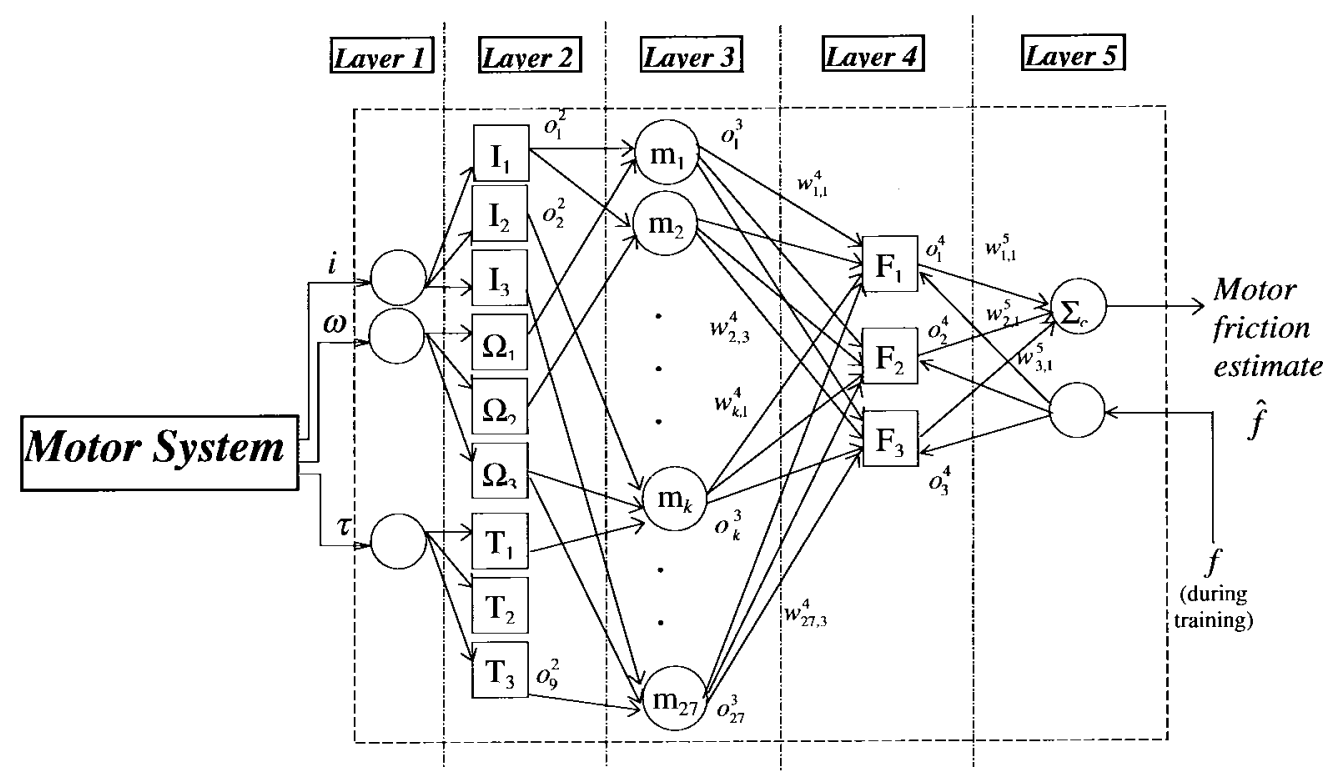

Fig. 2. The FFD structure.

Furthermore, the effects of incipient motor friction faults are highly coupled with effects of load torque. The aim of this paper is to estimate motor friction based on appropriate measurements.

A three-phase induction motor simulation program, MotorSIM, is used to provide the experimental data for the motor under different operating conditions to evaluate NN/FZ motor fault detector [2], [39], [40]. Nonlinear effects, such as temperature and saturation, were also incorporated into the simulation model. The specifications of this motor are given in [2], [39], and [40]. The data consist of three-phase stator currents and rotor angular velocity acquired under variable motor friction and load torque values.

\section{B. Motor Friction Fault}

This paper uses one of the most common motor incipient faults, the motor friction fault, to demonstrate the NN/FZ technology for motor fault detection/diagnosis. To simplify our discussion without loss of generality, the friction fault is assumed balanced, i.e., the fault will not cause unbalanced effect on an individual phase so that we can use single- 


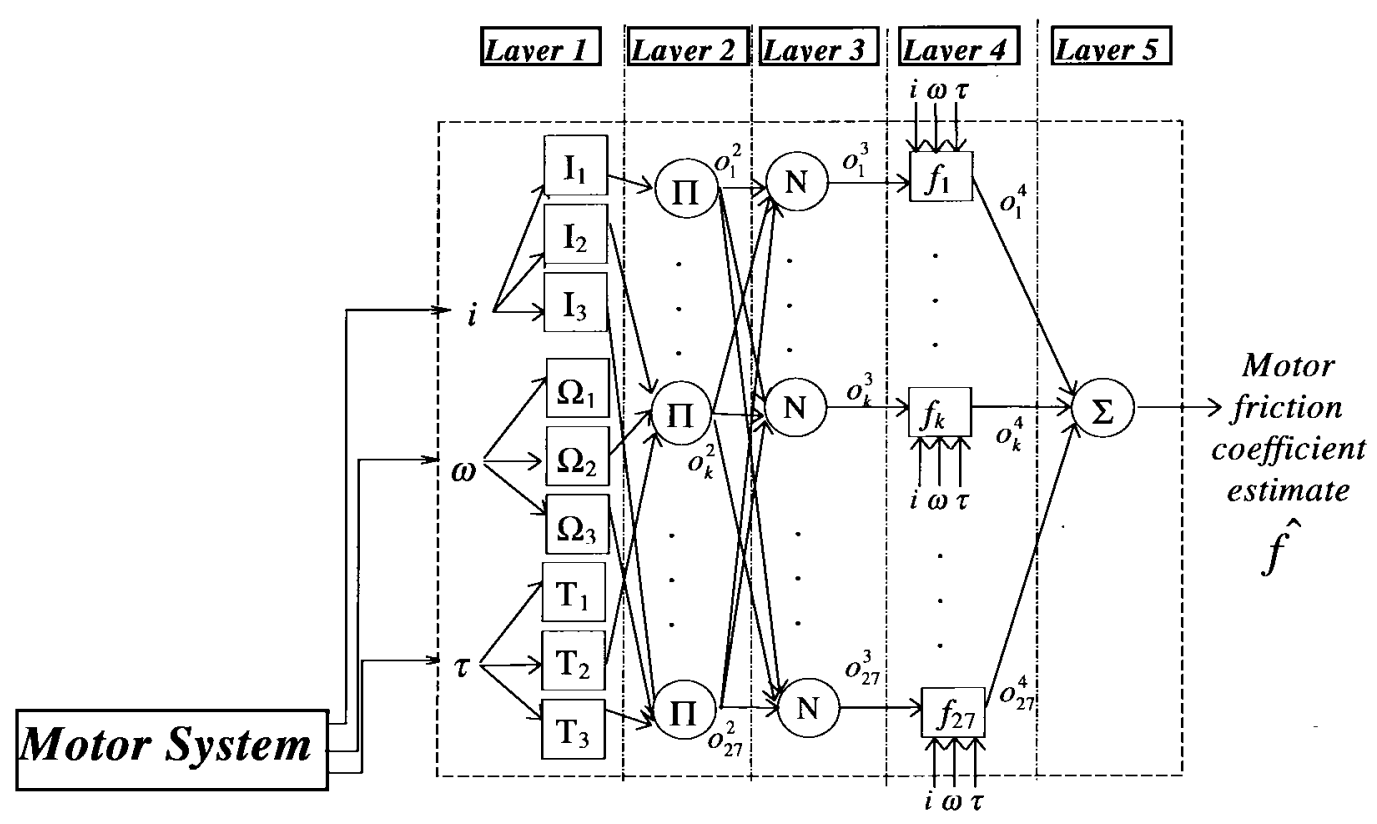

Fig. 3. AFD structure.

TABLE I

SPECIFICATIONS OF THE FUZZY INFERENCE Systems FOR AFD AND FFD

\begin{tabular}{l|l|l}
\hline & \multicolumn{1}{|c}{ FFD } & \multicolumn{1}{c}{ AFD } \\
\hline Membership functions & Gaussian & Gaussian \\
\hline Parameterization & Mean, variance & Mean, variance \\
\hline Fuzzy intersection & Minimum & Multiplication \\
\hline Fuzzy inference & Correlation-minimum & Correlation-product \\
operator & (Mamdani's Operator) & (Larsen's Operator) \\
\hline Aggregation & Addition & Addition \\
\hline Defuzzification & Center of area & Weighted average \\
& &
\end{tabular}

phase measurement to represent a three-phase case. As the motor ages, a bearing will change shape due to an imbalance of the motor or just fragmentation of the bearing itself; the lubricant will turn crisp and increase friction. These deformations eventually contribute to failures in other parts of the motor. The study in [41] provides an overview of the types of antifriction bearings most commonly used, factors affecting bearing life, and the relationship between the motor friction and temperature rise in induction motors. In the MotorSim, rotor angular velocity $\omega$ is described by the differential equation

$$
T_{e}=j \dot{\omega}+f \omega+\tau
$$

where $J$ is the moment of inertia for the rotor, $\omega$ is the rotor speed, $f$ is the friction coefficient of the rotor, $\tau$ is the rotor torque of the induction motor, and $T_{e}$ is the external (or load) torque. To simulate motor friction effect, the value of $f$ is increased. Ideal condition with no motor friction would imply $f=0$.

It is noted that the relation given in (1) is for the simulation model of the motor system, and provides the means for acquiring simulation data. However, the fault detection/diagnosis scheme is not dependent on the knowledge of this model; it is assumed that no a priori knowledge about the torque-speed relation of the system exists. This nonmodel-based approach to fault detection supports application of the technique to different motor systems without the requirement of precise model knowledge.

\section{Motor Fault Detector Structures}

Successful fault detection/diagnosis relies on the appropriate information being monitored. If the motor develops an incipient fault, we need to use the monitored information to detect the fault and determine its severity. It was previously demonstrated in [42] that, for a balanced friction fault, monitoring stator currents and rotor speed could lead to successful fault detection/diagnosis. In the presence of varying load situations, however, it is observed that the impact of load on motor current and speed is similar to that of the motor friction. For example, an increase in motor friction increases the current, and decreases the speed, which is similar to the effect of an increase in load. Therefore, if information about load is not included in our fault detection scheme during varying load torque operation, changes in load torque may lead to incorrect fault detection/diagnosis results. This paper employs two NN/FZ systems, which perform motor fault detection under different load conditions, and 
TABLE II

Summary of General Specifications of the Utilized Architectures

\begin{tabular}{c|c|c|c|c|c|c|c}
\hline $\begin{array}{c}\text { Fault Detector } \\
\text { Type }\end{array}$ & $\begin{array}{c}\text { Adaptive FIS } \\
\text { Type }\end{array}$ & $\begin{array}{c}\text { Adaptive } \\
\text { Architecture }\end{array}$ & $\begin{array}{c}\text { Algorithmic } \\
\text { Leaming } \\
\text { Structure }\end{array}$ & $\begin{array}{c}\text { Partition Of } \\
\text { Spaces }\end{array}$ & $\begin{array}{c}\text { Required Initial } \\
\text { Knowledge }\end{array}$ & $\begin{array}{c}\text { Structural } \\
\text { Change }\end{array}$ & $\begin{array}{c}\text { Extracted } \\
\text { Knowledge } \\
\text { Type }\end{array}$ \\
\hline AFD & ANFIS & $\begin{array}{c}\text { Multilayer } \\
\text { feedforward } \\
\text { network }\end{array}$ & $\begin{array}{c}\text { Hybrid; } \\
\text { supervised } \\
\text { (gradient descent } \\
\text { and LSE) }\end{array}$ & $\begin{array}{c}\text { Adaptive } \\
\text { fuzzy grid }\end{array}$ & $\begin{array}{c}\text { Numerical data or } \\
\text { Takagi-Sugeno type } \\
\text { fuzzy rules }\end{array}$ & No & $\begin{array}{c}\text { Takagi- } \\
\text { Sugeno type } \\
\text { fuzzy rules }\end{array}$ \\
\hline FFD & FALCON & $\begin{array}{c}\text { Multilayer } \\
\text { feedforward } \\
\text { network }\end{array}$ & $\begin{array}{c}\text { Hybrid; } \\
\text { supervised and } \\
\text { unsupervised }\end{array}$ & $\begin{array}{c}\text { Adaptive } \\
\text { fuzzy grid }\end{array}$ & $\begin{array}{c}\text { Numerical data or } \\
\text { fuzzy IF-THEN rules }\end{array}$ & Yes & $\begin{array}{c}\text { Fuzzy IF- } \\
\text { THEN rules }\end{array}$ \\
\hline
\end{tabular}

are able to extract heuristics for the fault detection process. The conceptual diagram of the NN/FZ motor fault detection structure is depicted in Fig. 1.

The fault detector is a fuzzy inference system implemented on an adaptive network structure. The stator current $i$, rotor speed $\omega$, and load torque $\tau$ of the motor are the inputs of the fault detector, and are used to estimate the motor friction $f$. The universes of discourse of the current, speed, and load torque are defined as

$$
\begin{aligned}
\boldsymbol{I} & =\left\{i \mid i_{l} \leq i \leq i_{u}\right\} \\
\boldsymbol{\Omega} & =\left\{\omega\left|\omega_{l} \leq \omega \leq \omega_{u}\right|\right. \\
\boldsymbol{T} & =\left\{\tau \mid 0 \leq \tau \leq \tau_{u}\right\}
\end{aligned}
$$

respectively. Here, $i_{l}, i_{u}, \omega_{l}, \omega_{\subset} \Re$ are constants representing the upper and lower bounds for the feasible current and speed measurements in the operating range, and $\tau_{u} \in \Re$ is the upper limit of feasible values for load torque in the operating range under consideration. Three fuzzy sets are defined on each of the input spaces, corresponding to low, medium, and high for each variable, and labeled $I_{k}, \Omega_{k}$, and $T_{k}$, respectively, with $1 \leq k \leq 3$. The input space $\boldsymbol{X}$ is defined as the Cartesian product of the current, speed, and load torque spaces

$$
X=I \times \Omega \times T .
$$

The output of the fault detector is the friction coefficient $f$. The output space $\boldsymbol{Y}$ of the fault detector is defined as

$$
\boldsymbol{Y}=\boldsymbol{F}=\left\{f \mid 0 \leq f \leq f_{u}\right\}
$$

where $f_{u} \in \Re$ represents the upper limit of the friction coefficient. The fault detection process may be viewed as a mapping from the input space to the output space, which maps the operating current, speed, and load torque to motor friction.

\section{NN/FZ Motor Fault Detection ARChitectures}

\section{A. FFD}

The FFD structure is based on the model of the FALCON proposed in [17]. This is an adaptive fuzzy inference system constructed automatically via training with system data. The FFD architecture is a five-layered feedforward networkbased fuzzy inference system. The connectionist structure is isomorphic to an $\mathrm{NN}$, with layers corresponding to input states, decision states, and hidden layers that substitute the fuzzy inference engine in terms of aggregation, defuzzification, and decision making [43]. Fuzzy rules of the motor fault detection/diagnosis problem and membership functions used in these rules are implemented in the hidden-layer nodes. The membership functions are parameterized with center and width values [17]. The FFD structure for the motor fault detection/diagnosis problem is shown in Fig. 2. The structure mimics a fuzzy inference system; the calculations are carried out in a distributed manner.

The FFD training procedure includes both unsupervised (self-organized) and supervised learning schemes. The unsupervised training provides a priori fuzzy partitioning of the input space by defining the initial membership functions and finding the existence of the rules. The second part of the training is an optimal adjustment phase of the membership parameters. The training minimizes an error function, with given input, desired output, and fuzzy partitions of the input and output spaces. Different on-line structure/parameter learning algorithms based on the FALCON were also proposed in [43].

\section{B. $A F D$}

The AFD is based on Jang's ANFIS [44], which is another fuzzy inference system implemented on the architecture of a five-layer feedforward network. By using a hybrid learning procedure, the AFD can construct an input-output mapping based on both human knowledge (in the form of Takagi-Sugeno-type if-then rules) and input-output data observations. Takagi-Sugeno-type if-then rules used in this paper have fuzzy antecedents, but a crisp consequence, which is a linear combination of the input values, e.g.,

$$
\begin{aligned}
\left(i \in I_{1}\right) \Lambda\left(\omega \in \Omega_{1}\right) \Lambda(\tau & \left.\in T_{1}\right) \\
& \Rightarrow\left(f=a_{0}+a_{l} i+a_{2} \omega+a_{3} \tau\right) .
\end{aligned}
$$

The membership functions that form the antecedents, as well as the functions that form the consequence parts, are parameterized using a method similar to the one in the FFD. The AFD structure for the motor fault detection/diagnosis problem is shown in Fig. 3.

The hybrid learning procedure is composed of a forward pass and a backward pass. In the forward pass, the antecedent parameters are fixed and the consequence parameters are optimized via least-squares estimation. Once the optimum consequence parameters are found, the backward pass stage starts. In this stage, gradient descent is used to optimally adjust the antecedent membership parameters corresponding 


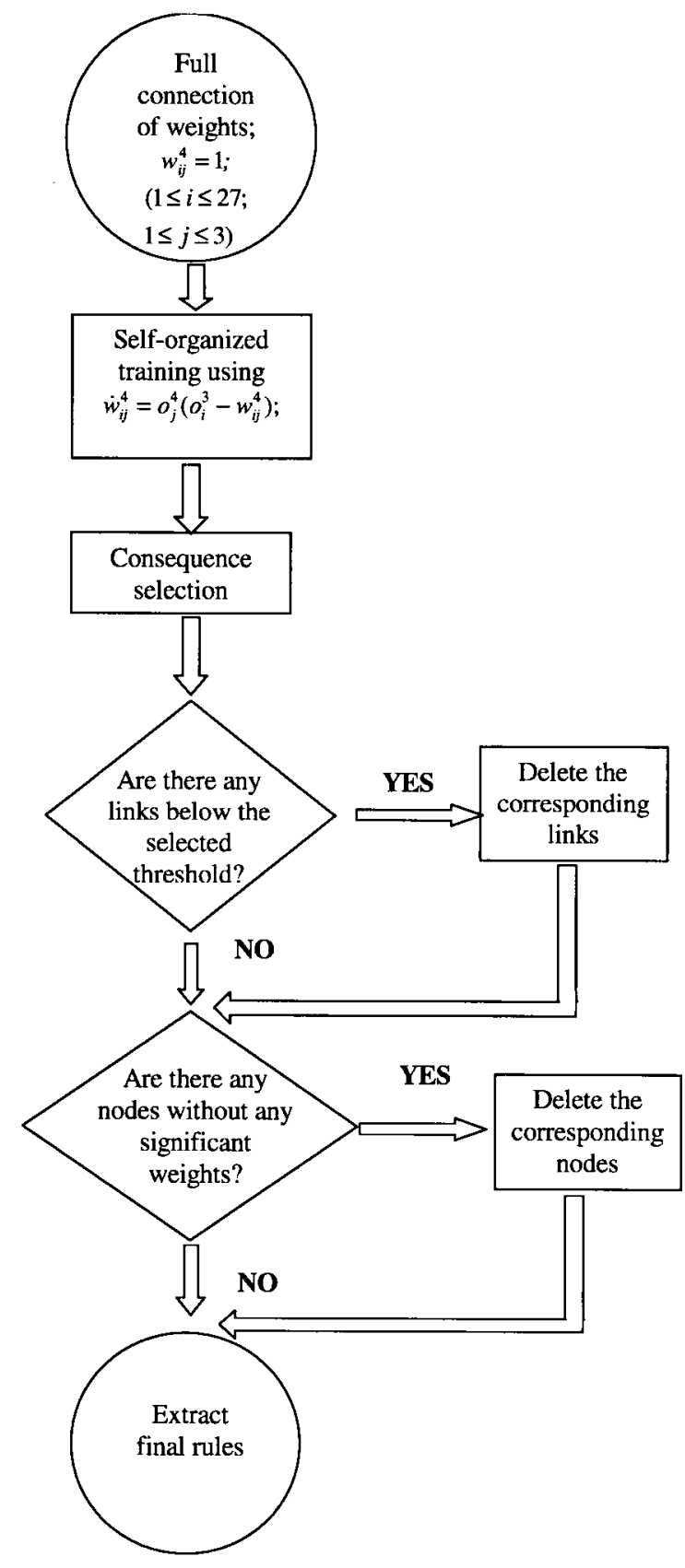

Fig. 4. Flow chart for rule revision and extraction from the FFD.

to the fuzzy sets in the input domain. The output of the $\mathrm{NN}$ is calculated by fixing the consequence parameters to the values found in the forward pass. The output error of the $\mathrm{NN}$ is then used to adapt the antecedent parameters using a standard backpropagation algorithm. Similar structures have also been used in various applications, including gain scheduling [45] and system parameter identification [46].

\section{COMPARISON OF AFD AND FFD}

\section{A. Fuzzy Inference System Specifications}

As mentioned previously, the NN/FZ fault detector structures can be viewed as fuzzy inference systems built on

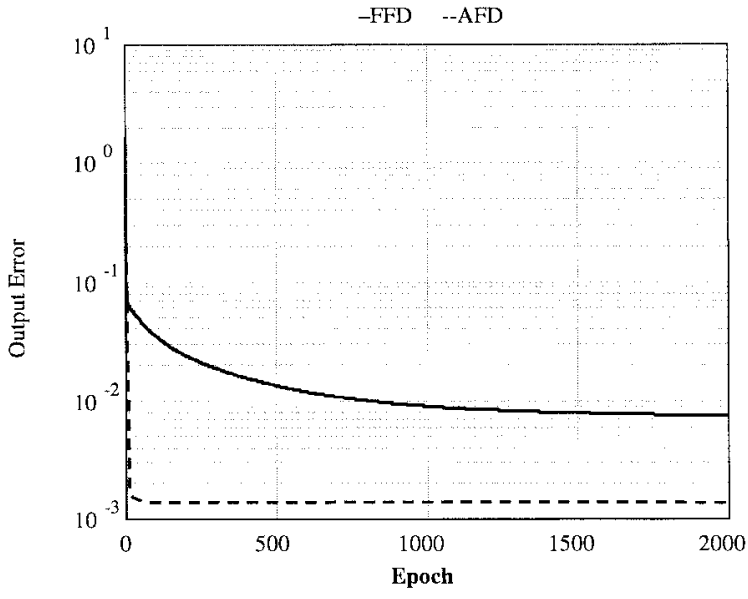

Fig. 5. Output error versus epoch for FFD and AFD for motor friction.

TABLE III

EXtracted RULEs for Motor Friction from the FFD

\begin{tabular}{llll}
\hline If Current & $\begin{array}{l}\text { and Speed } \\
\text { Is }\end{array}$ & $\begin{array}{l}\text { And Load } \\
\text { Is }\end{array}$ & $\begin{array}{l}\text { then Motor Friction } \\
\text { is }\end{array}$ \\
\hline Low & Medium & Low & Medium \\
Low & Medium & Medium & Low \\
Low & Medium & High & Low \\
Low & High & Low & Medium \\
Low & High & Medium & Low \\
Low & High & High & Low \\
Medium & Low & Low & High \\
Medium & Low & Medium & High \\
Medium & Medium & Low & High \\
Medium & Medium & Medium & Medium \\
Medium & Medium & High & Low \\
Medium & High & Low & Medium \\
Medium & High & Medium & Low \\
Medium & High & High & Low \\
High & Low & Low & High \\
High & Low & Medium & High \\
High & Medium & Low & High \\
High & Medium & Medium & High \\
\hline & & &
\end{tabular}

adaptive architectures. The specifications of the fuzzy inference systems underlying the NN/FZ structures are given in Table I, for the AFD and the FFD. The membership functions mentioned in the table correspond to the input and output membership functions for the FFD, and the input membership functions only, for the AFD.

\section{B. Architectural Specifications}

This section gives the specifications of the adaptive structures on which the fuzzy inference systems were built. A summary of the AFD and FFD in terms of learning algorithms, initial knowledge requirements, extracted knowledge types, domain partitioning, and rule structuring and revising are given in Table II. In the table, Structural Change indicates that 
TABLE IV

EXTRACTED RULES FOR Motor Friction From the AFD

\begin{tabular}{|c|c|c|c|c|c|c|}
\hline \multirow{2}{*}{$\begin{array}{l}\text { If } \\
\text { Current } \\
\text { is }\end{array}$} & \multirow{2}{*}{$\begin{array}{l}\text { and } \\
\text { Speed } \\
\text { is }\end{array}$} & \multirow{2}{*}{$\begin{array}{l}\text { and } \\
\text { Torque } \\
\text { is }\end{array}$} & $\begin{array}{l}\text { Then } \\
\text { Motor Friction = }\end{array}$ & \multicolumn{2}{|c|}{$a_{0}+a_{1 .} i+a_{2} \omega+a_{3} \tau$} & \multirow[b]{2}{*}{$a_{3}$} \\
\hline & & & $a_{0}$ & $a_{L}$ & $a_{2}$ & \\
\hline Low & Low & Low & -0.000080 & -0.002066 & 0.000009 & -0.000006 \\
\hline Low & Low & Medium & 0.000064 & 0.001869 & 0.000017 & 0.000005 \\
\hline Low & Low & High & 0.000015 & 0.000731 & 0.000017 & 0.000002 \\
\hline Low & Medium & Low & -0.007231 & 0.000715 & -0.004680 & -0.000142 \\
\hline Low & Medium & Medium & 0.006610 & 0.000270 & -0.003254 & 0.000077 \\
\hline Low & Medium & High & 0.001077 & -0.000616 & 0.001091 & 0.000012 \\
\hline Low & High & Low & 0.003800 & -0.000040 & -0.003413 & 0.001234 \\
\hline Low & High & Medium & -0.009238 & 0.000105 & -0.001410 & -0.000276 \\
\hline Low & High & High & -0.000954 & 0.000175 & -0.000913 & -0.000010 \\
\hline Medium & Low & Low & 0.000385 & 0.004829 & -0.001178 & 0.000017 \\
\hline Medium & Low & Medium & -0.000787 & -0.001354 & -0.002979 & -0.000012 \\
\hline Medium & Low & High & -0.000809 & 0.002709 & -0.001996 & -0.000003 \\
\hline Medium & Medium & Low & -0.007552 & 0.000639 & -0.006557 & -0.000116 \\
\hline Medium & Medium & Medium & 0.008476 & -0.000172 & -0.001871 & 0.000123 \\
\hline Medium & Medium & High & -0.001331 & 0.000268 & -0.003028 & -0.000007 \\
\hline Medium & High & Low & 0.005266 & -0.001111 & 0.004863 & 0.000084 \\
\hline Medium & High & Medium & -0.002410 & 0.000164 & -0.006782 & -0.000021 \\
\hline Medium & High & High & 0.000514 & 0.000680 & 0.000909 & 0.000009 \\
\hline High & Low & Low & 0.000361 & 0.000343 & 0.000614 & 0.000006 \\
\hline High & Low & Medium & 0.000582 & 0.000573 & -0.003838 & 0.000010 \\
\hline High & Low & High & 0.007055 & -0.000095 & -0.004106 & 0.000107 \\
\hline High & Medium & Low & -0.000208 & -0.002087 & -0.002117 & -0.000007 \\
\hline High & Medium & Medium & -0.001226 & 0.001379 & -0.004767 & -0.000010 \\
\hline High & Medium & High & -0.001882 & -0.000714 & -0.003380 & -0.000025 \\
\hline High & High & Low & 0.000085 & 0.003685 & -0.000007 & 0.000010 \\
\hline High & High & Medium & -0.000054 & -0.003187 & -0.000120 & -0.000008 \\
\hline High & High & High & -0.000018 & -0.000327 & -0.000021 & -0.000001 \\
\hline
\end{tabular}

the architecture enables a change in the rule structure, i.e., a combination of different antecedents with different consequences, during the evolution of the fuzzy inference system. A fuzzy inference system (with noninteractive fuzzy information) can be viewed as a partition in the multidimensional feature space, where the number of partitions in each dimension corresponds to the number of fuzzy sets and the corresponding membership functions that are defined in that dimension. An Adaptive Partitioning means that the parameters of the fuzzy sets defined in each dimension are allowed to change in an effort to optimize the network with respect to an error measure. Required Initial Knowledge is the knowledge that is externally supplied to the architecture initially. Extracted Knowledge stands for the knowledge that can be transparently acquired from the structure after training.

1) Knowledge Extraction: A clear advantage of the FFD system is that knowledge in terms of fuzzy if-then rules can be extracted from the system in a straightforward manner. The layer-4 weight matrix of the FFD, after the self-organized training, provides the necessary structural information. The knowledge acquired from the AFD system is not as transparent as the FFD. In the rules in the AFD, the estimate of function, which is the consequence part of the rules, is in the form of a weighted average, not a fuzzy proposition. Therefore, it is not obvious how to extract heuristic fuzzy if-then rules from the AFD.

The unsupervised training in the FFD leads to structuring of rules. Using the weight update method given in [17], the layer-
4 connection weights are updated so that the final connections provide the strongest antecedent-consequence relations, which are then selected as the rules. The weights at layer- 4 connect the $i$ th antecedent node to the $j$ th consequence node. Higher connection weights can be interpreted as implying that the corresponding rules have larger impact in the fault diagnosis process. After this elimination, if there exist no connections from a layer-4 node to any layer-3 node, then this layer-4 node is eliminated as it has no impact on the output. The flow chart of the rule structuring and modification procedure is given in Fig. 4.

\section{RESUlts OF InCIPIENT MOTOR FAUlT DETECTION USING FFD AND AFD}

The performances of both FFD and AFD architectures for the motor fault detection evaluated in terms of accuracy in fault detection and extracted knowledge quality are discussed in this section.

\section{A. Fault Detection Accuracy}

Fault detection accuracy is calculated in terms of the rms and maximum error for both the FFD and AFD. The output error is defined as $\varepsilon_{i}=\left|f_{i}-\hat{f}_{i}\right|$, where $i$ is the experiment index, $f_{i}$ are the actual values of motor friction (or damping) coefficient, and $\hat{f}_{i}$ are the fault detector estimates for the motor friction coefficient. The error vector is

$$
\varepsilon=\left[\begin{array}{llll}
\varepsilon_{1} & \varepsilon_{2} & \cdots & \varepsilon_{N}
\end{array}\right]^{T}
$$




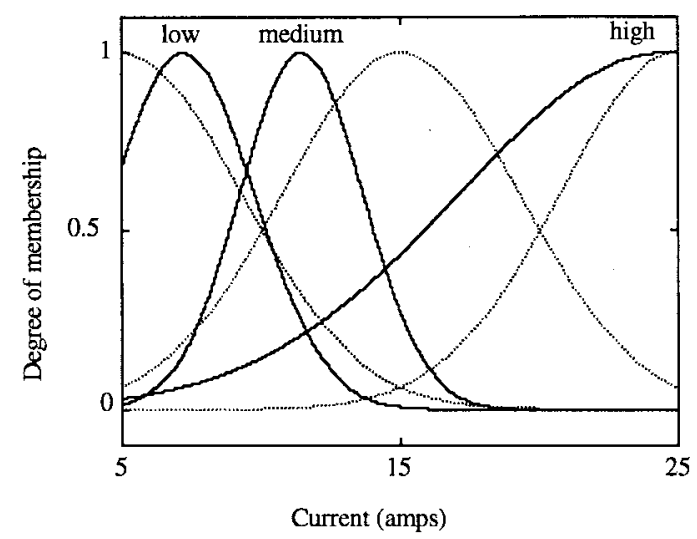

(a)

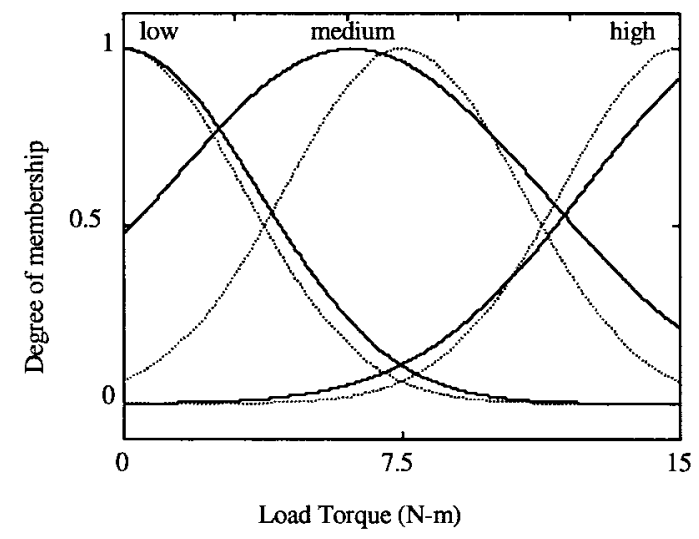

(c)

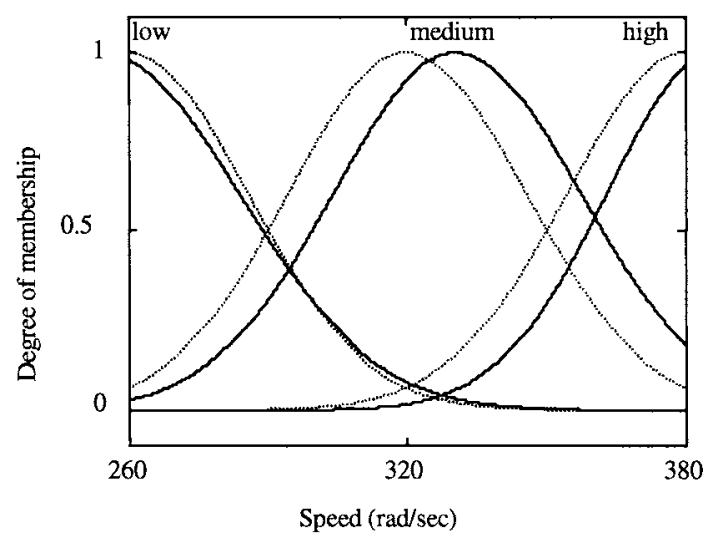

(b)

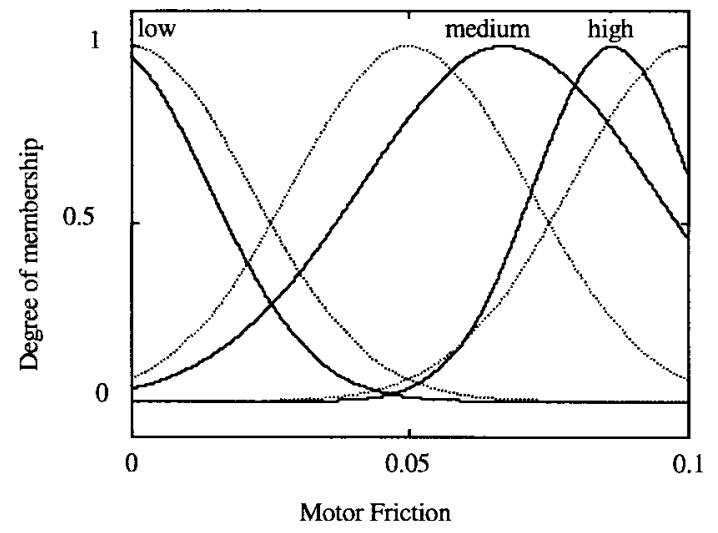

(d)

Fig. 6. The final membership functions for (a) current, (b) speed, (c) load, and (d) motor friction for friction fault detection with the FFD.

where $N$ is the total number of data observations. We define the two-norm error $\|\varepsilon\|_{2}$ and the $\infty$-norm error $\|\varepsilon\|_{\infty}$ as

$$
\|\varepsilon\|_{2}=\left(\sum_{i=1}^{N} \varepsilon_{i}^{2}\right)^{1 / 2} \quad\|\varepsilon\|_{\infty}=\max _{i}\left(\left|\varepsilon_{i}\right|\right)
$$

respectively. Following these definitions, Fig. 5 presents the rms output error defined as $N^{-1 / 2}\|\varepsilon\|_{2}$, versus training epochs for the motor friction, for FFD and AFD. It is observed that both NN/FZ fault detectors can provide accurate fault detection/diagnosis. Convergence speed depends on certain learning coefficients used in the process of supervised learning of both NN/FZ architectures. There is a visible difference in the convergence speed, yet the performance of the final fault detectors is about the same. The convergence speed, as well as performance, may be improved further by fine tuning several coefficients related to the training of the fault detectors.

These two fault detector structures are compared in terms of $\|\varepsilon\|_{\infty}$ and $\|\varepsilon\|_{2}$. The AFD performs better with regard to $\infty$ - and two-norm output error, with $\| \varepsilon=0.00171$ and $\|\varepsilon\|_{2}=0.00113$. For the FFD, $\|\varepsilon\|_{\infty}=0.00953$, and $\| \varepsilon=0.00872$. The orders of the two-norm and $\infty$-norm errors are observed to be smaller for the AFD.

\section{B. Extracted Knowledge}

As mentioned previously, knowledge is extracted from the FFD in terms of if-then fuzzy rules. The list of the rules extracted from the FFD is given in Table III for motor friction. Even though no a priori knowledge was incorporated into the fault detection/diagnosis process, the rules are observed to be in agreement with our knowledge about the system, which states that the impact of motor friction on stator current and rotor speed is similar to that of the load. The extracted rules are consistent with our expectations. For example, for low-current and high-speed propositions for current and speed, there are two rules in our database in the form

$$
\begin{aligned}
& \left(i \in I_{1}\right) \Lambda\left(\omega \in \Omega_{3}\right) \Lambda\left(\tau \in T_{2}\right) \Rightarrow\left(f \in F_{1}\right) \\
& \left(i \in I_{1}\right) \Lambda\left(\omega \in \Omega_{3}\right) \Lambda\left(\tau \in T_{1}\right) \Rightarrow\left(f \in F_{2}\right) .
\end{aligned}
$$

The extracted information suggests that low current and high speed, when combined with medium load, would imply a low friction condition. However, the same conditions for current and speed, when combined with low load, imply a medium friction condition.

On the other hand, the AFD presents information about the output space in the form linear functions of inputs, rather than like the extracted information in Table III. Thus, the AFD is not as transparent as the FFD to extract information in the form of if-then fuzzy rules. The list of Takagi-Sugeno rules extracted from the AFD is given in Table IV for the motor friction.

The final membership functions for the current, speed, load torque, and friction coefficient are given in Fig. 6 for the FFD. The final input space membership functions for the AFD are 


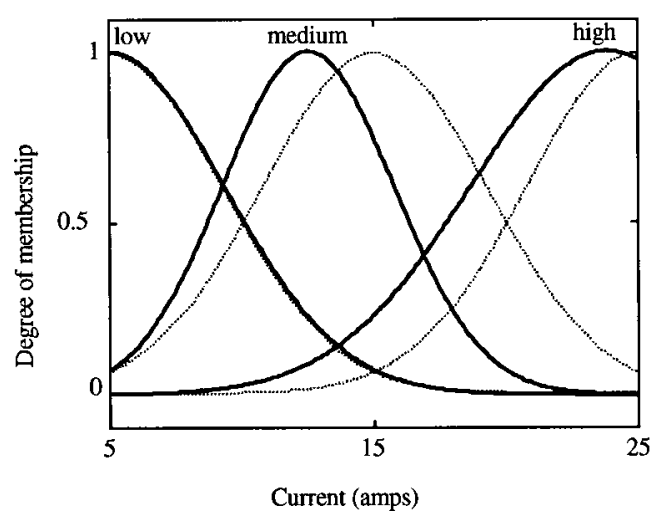

(a)

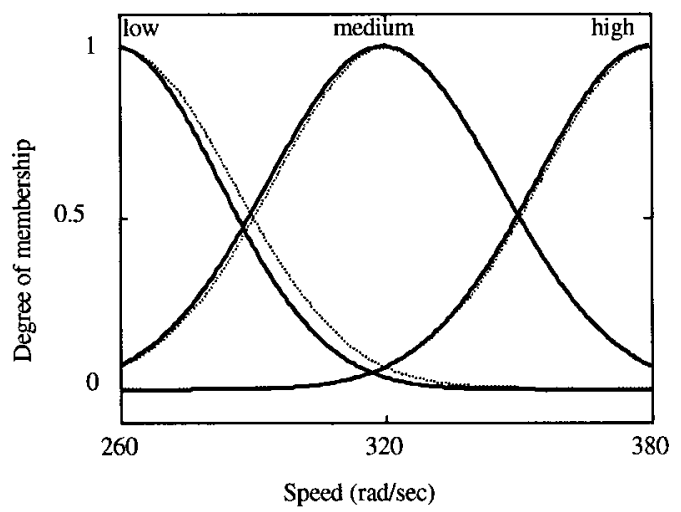

(b)

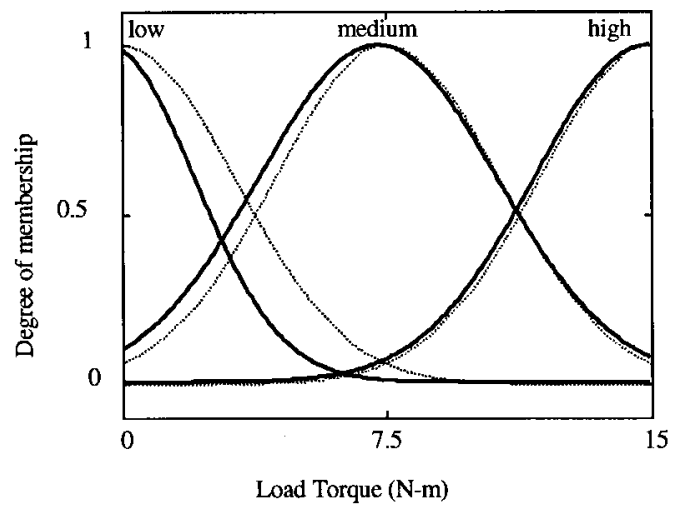

(c)

Fig. 7. Final membership functions for (a) current, (b) speed, and (c) load for friction fault detection with the AFD.

given in Fig. 7. The initial membership functions are also plotted in Figs. 6 and 7, with dashed lines. After the initial training step of the AFD, which is the optimization of the consequence parameters, the system adapts such that the motor friction estimate is significantly close to the actual motor friction with regard to two-norm. Therefore, in the backward pass, which uses gradient descent to adapt membership function shapes, the network structure does not change significantly. Hence, the output error converges without a large change in the input membership mean and variance values.

\section{Discussion AND CONCLUSIONS}

This paper has described and illustrated the application of two popular NN/FZ systems, the AFD and the FFD, for motor fault detection/diagnosis. Both structures can provide good fault detection/diagnosis under varying load torque, with the results of the AFD being slightly more accurate. Yet, from the FFD, consistent heuristic information can be extracted in terms of fuzzy if-then rules, which is probably one of the main advantages of the structure. However, for the specific case considered, the fault detection scheme is slower in convergence when compared to the AFD. Furthermore, the initial unsupervised pretraining is mandatory for the preliminary structuring of the fuzzy inference system. The AFD, on the other hand, is faster in convergence. Furthermore, it provides better results when applied without any pretraining. However, the extracted knowledge in the AFD is not as easy and straightforward as in the case of the FFD due to the tradeoff made by utilizing linear output space functions (and using least-squares estimation in training). Therefore, the final knowledge extracted is not in the form of pure heuristic rules, i.e., rules that can be expressed purely by linguistic terms. This reduces the effectiveness of the fault detector, because it is hard to provide heuristic interpretation to the solution. An ideal neural/fuzzy fault detector architecture would combine the rule extraction power of the FFD and the speed and accuracy of the AFD, for high-dimensional fault detection problems. These results suggest new and promising research areas utilizing NN/FZ systems in induction motor fault detection and diagnosis.

\section{REFERENCES}

[1] M. Y. Chow, R. N. Sharpe, and J. C. Hung, "On the application and design of artificial neural networks for motor fault detection-Part 1," IEEE Trans. Ind. Electron., vol. 40, pp. 181-188, Apr. 1993.

[2] M. Y. Chow, Methodologies of Using Artificial Neural Network and Fuzzy Logic Technologies for Motor Incipient Fault Detection. Singapore: World Scientific, 1997.

[3] K. F. Martin, "Review by discussion of condition monitoring and fault diagnosis in machine tools," Int. J. Mach. Tools Manuf., vol. 34, no. 4, pp. 527-551, 1994.

[4] W. R. Finley and R. R. Borke, "Troubleshooting motor problems," IEEE Trans. Ind. Applicat., vol. 30, pp. 1383-1939, Sept./Oct. 1992.

[5] A. K. Sood, A. A. Fahs, and N. A. Henein, "Engine fault analysis, part I: Statistical methods," IEEE Trans. Ind. Electron., vol. 32, pp. 294-300, Nov. 1985.

[6] _Engine fault analysis part II: Parameter estimation approach," IEEE Trans. Ind. Electron., vol. 32, pp. 301-307, Nov. 1985. 
[7] R. Isermann, "Process fault detection based on modeling and estimation methods-a survey," Automatica, vol. 20, pp. 387-404, 1984.

[8] R. Isermann and B. Freyermuth, "Process fault diagnosis based on process model knowledge-Part 1," J. Dynam. Syst., Meas., Contr., vol. 113, pp. 620-626, 1991

[9] M. Y. Chow and S. O. Yee, "Methodology for on-line incipient fault detection in single phase squirrel cage induction motors using artificial neural networks," IEEE Trans. Energy Conversion, vol. 6, pp. 536-545, Sept. 1991

[10] R. Maier, "Protection of squirrel-cage induction motor utilizing instantaneous power and phase information," IEEE Trans. Ind. Applicat., vol. 28, pp. 376-380, Mar./Apr. 1992.

[11] A. H. Bonnet and G. C. Soukup, "Cause and analysis of stator and rotor failures in three-phase induction motors," IEEE Trans. Ind. Applicat., vol. 28, pp. 921-937, July/Aug. 1992.

[12] D. J. T. Siyambalapitiya and P. G. Mclaren, "Reliability improvement and economic benefits of on-line monitoring systems for large induction machines," IEEE Trans. Ind. Applicat., vol. 26, pp. 1018-1025, Nov./Dec. 1990.

[13] K. R. Cho, J. H. Lang, and S. Umans, "Detection of broken rotor bars using state and parameter estimation," IEEE Trans. Ind. Applicat., vol. 28, pp. 702-713, May/June 1992.

[14] S. Ansuj, F. Shokooh, and R. Schinziger, "Parameter estimation for induction machines based on sensitivity analysis," IEEE Trans. Ind. Applicat., vol. 25, pp. 1035-1040, Nov./Dec. 1989.

[15] P. V. Goode and M. Y. Chow, "Using a neural/fuzzy system to extract heuristic knowledge of incipient faults in induction motors: Part I-Methodology," IEEE Trans. Ind. Electron., vol. 42, pp. 131-138, Apr. 1995.

[16] J. R. Jang and C. T. Sun, "Neuro-fuzzy modeling and control," Proc. IEEE, vol. 83, pp. 378-406, Mar. 1995.

[17] C. T. Lin and C. G. Lee, "Neural-network-based fuzzy logic control and decision system," IEEE Trans. Comput., vol. 40, pp. 1320-1336, Dec. 1991.

[18] S. G. Kong and B. Kosko, "Adaptive fuzzy systems for backing up a truck and trailer," IEEE Trans. Neural Networks, vol. 3, pp. 211-223, Mar. 1992.

[19] J. Nie, "Constructing fuzzy model by self-organizing counter propagation network," IEEE Trans. Syst., Man, Cybern., vol. 25, pp. 963-970, June 1995.

[20] H. Ishibuchi, R. Fujioka, and H. Tanaka, "Neural networks that learn from fuzzy IF-THEN rules," IEEE Trans. Fuzzy Syst., vol. 1, pp. 85-97, May 1993.

[21] R. R. Yager, "Implementing fuzzy logic controllers using a neural network framework," Fuzzy Sets Syst., vol. 48, pp. 53-64, 1992.

[22] C. T. Lin and C. S. G. Lee, Neural Fuzzy Systems. Englewood Cliffs, NJ: Prentice-Hall, 1996.

[23] F. Filipetti, G. Franchescini, and C. Tassoni, "Neural networks aided online diagnostics of induction motor faults," IEEE Trans. Ind. Applicat., vol. 31, pp. 892-899, July/Aug. 1995.

[24] A. Bernieri, M. D'apuzzo, L. Sansone, and M. Savastano, "A neural network approach for identification and fault diagnosis on dynamic systems," IEEE Trans. Instrum. Meas., vol. 43, pp. 867-873, Dec. 1994

[25] I. Alguindigue, A. L. Buczak, and R. E. Ulrig, "Monitoring and diagnosing element bearing faults using neural networks," IEEE Trans. Ind. Electron., vol. 40, pp. 209-217, Apr. 1993.

[26] R. Isermann and B. Freyermuth, "Process fault diagnosis based on process model knowledge-Part 2," J. Dynam. Syst., Meas., Contr., vol. 113, pp. 627-633, 1991

[27] K. S. Narendra and K. Parthasarathy, "Identification and control of dynamic systems using neural networks," IEEE Trans. Neural Networks, vol. 1, pp. 4-27, Mar. 1991.

[28] S. R. Chu, R. Shoureshi, and M. Tenorio, "Neural networks for system identification," IEEE Contr. Syst. Mag., vol. 10, pp. 31-35, Apr. 1990.

[29] J. Penman and C. M. Yin, "The application of artificial neural networks in identification of faults in induction machines," in Proc. ICEM'92, 1992.

[30] R. N. Sharpe, M. Y. Chow, S. Briggs, and L. Windigland, "Methodology using fuzzy logic to optimize feedforward artificial neural network configurations," IEEE Trans. Syst., Man, Cybern., vol. 24, pp. 760-767, May 1994.

[31] Y. Chen, "A fuzzy decision system for fault classification under high levels of uncertainty," Trans. ASME, vol. 117, pp. 108-115, 1995.

[32] L. Zeng and H. P. Wang, "Machine fault classification: A fuzzy set approach," Int. J. Adv. Manuf. Technol., vol. 6, pp. 83-94, 1991.

[33] I. Gazdik, "Fault diagnosis and prevention by fuzzy sets," IEEE Trans. Rel., vol. R-34, pp. 382-388, Oct. 1985.
[34] C. Li and C.-H. Wu, "Automatically generated rules and membership functions for a neural fuzzy-based fault classifier," in Proc. Midwest Symp. Circuits and Systems, 1994, vol. 2, pp. 1377-1380.

[35] N. Maruyama, M. Benouarets, and A. L. Dexter, "Detecting faults in nonlinear dynamic systems using static neuro-fuzzy models," in Inst. Elect. Eng. Colloquium, 1995, no. 79, pp. 8/1-8/10

[36] P. M. Frank, "Residual evaluation for fault diagnosis based on adaptive fuzzy thresholds," in Inst. Elect. Eng. Colloq., 1995, no. 79, pp. 4/1-4/11.

[37] R. J. Kuo, "Intelligent diagnosis for turbine blade faults using artificial neural networks and fuzzy logic," Eng. Applicat. Artif. Intell., vol. 8, pp. 25-34, 1995.

[38] J. Zhang and A. Julian, "On-line process fault diagnosis using fuzzy neural networks," Intell. Syst. Eng., vol. 3, no. 1, pp. 37-47, 1994.

[39] M. Y. Chow, S. Altug, and B. Li, "Motor system time domain fast prototype software simulation," in Proc. Nat. Symp. Electric Machines, 1997.

[40] M. Y. Chow, "Motor fault detection and diagnosis," IEEE Ind. Electron. Newslett., vol. 44, no. 4, 1997.

[41] B. Maru and P. A. Zotos, "Anti-friction bearing temperature rise for NEMA frame motors," IEEE Trans. Ind. Applicat., vol. 25, pp. 883-888, Sept./Oct. 1989.

[42] P. V. Goode and M. Y. Chow, "Using a neural/fuzzy system to extract heuristic knowledge of incipient faults in induction motors: Part II-Application," IEEE Trans. Ind. Electron., vol. 42, pp. 139-146, Apr. 1995.

[43] C.-T. Lin, C.-J. Lin, and C. G. Lee, "Fuzzy adaptive learning control network with on-line neural learning," Fuzzy Sets Syst., vol. 71, no. 1, pp. $25-45,1995$

[44] J. R. Jang, "ANFIS: Adaptive-network-based fuzzy inference system," IEEE Trans. Syst., Man, Cybern., vol. 23, pp. 665-685, May/June 1993.

[45] J. R. Jang and N. Gulley, "Gain scheduling based fuzzy controller design," in Proc. Int. Joint Conf. NAFIPS, 1994, vol. 1, pp. 101-105.

[46] C. T. Sun, "Rule-base structure identification in an adaptive-networkbased fuzzy inference system," IEEE Trans. Fuzzy Syst., vol. 2, pp. 64-73, Feb. 1994

Sinan Altug was born in Ankara, Turkey. He received the B.S. and M.S degrees in electrical engineering in 1992 and 1994, respectively, from Middle East Technical University, Ankara, Turkey, and the M.B.A. degree in 1994 from Bilkent University, Ankara, Turkey. He is currently working toward the Ph.D. degree in the Department of Electrical and Computer Engineering, North Carolina State University, Raleigh

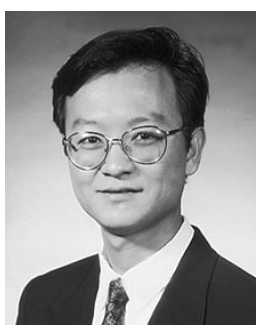

Mo-Yuen Chow (S'81-M'82-SM'93) received the B.S. degree from the University of Wisconsin, Madison, and the M.Eng. and Ph.D. degrees from Cornell University, Ithaca, NY, in 1982, 1983, and 1987, respectively.

Following receipt of the Ph.D. degree, he joined the faculty of North Carolina State University, Raleigh, where he is currently a Professor in the Department of Electrical and Computer Engineering. He has also worked as a Consultant for various companies. His core technology is diagnosis and control, artificial neural networks, and fuzzy logic. He has served as a Principal Investigator in projects supported by federal agencies, companies, and research centers. He established the Advanced Diagnosis and Control Laboratory at North Carolina State University. He is the author of numerous published works, including one book, several book chapters, and over 70 journal and conference articles related to his research. He was President of the Triangle Area Neural Network Society during 1996-1998.

Dr. Chow is currently an Associate Editor of the IEEE TRAnsactions ON InDUSTRIal ElECTRONICS and served as a Guest Editor for the Special Issue on Application of Intelligent Systems to Industrial Electronics. He is an AdCom member of the IEEE Industrial Electronics Society, an AdCom member of the IEEE Neural Network Council, and Chairman of the IEEE Neural Network Council Regional Interest Group Committee. He is listed in Who's Who in Asian Americans. 


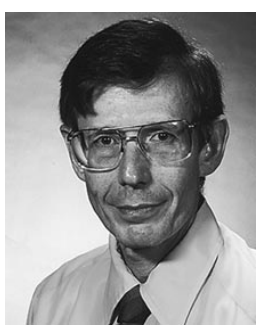

H. Joel Trussell (S'75-M'76-SM'91-F'94) received the B.S. degree from Georgia Institute of Technology, Atlanta, the M.S. degree from Florida State University, Tallahassee, and the Ph.D. degree from the University of New Mexico, Albuquerque, in 1967, 1968, and 1976, respectively.

In 1969, he joined the Los Alamos Scientific Laboratory, Los Alamos, NM, where he began working in image and signal processing in 1971. During 1978-1979, he was a Visiting Professor at Heriot-Watt University, Edinburgh, U.K., where he worked with both the university and with industry on image processing problems. In 1980, he joined the Electrical and Computer Engineering Department, North Carolina State University, Raleigh. During 1988-1989, he was a Visiting Scientist at Eastman Kodak Company, Rochester, NY. During 1997-1998, he was a Visiting Scientist at Color Savvy Systems, Springboro, $\mathrm{OH}$. His research has been in estimation theory and signal and image restoration.

Dr. Trussell is a Past Associate Editor of the IEEE Transactions on Acoustics, Speech, and Signal Processing and is currently an Associate Editor of the IEEE Signal Processing Letters. He was a Member and Past Chairman of the Image and Multidimensional Digital Signal Processing Committee of the IEEE Signal Processing Society. He was elected and served on the Board of Governors of the IEEE Signal Processing Society during 1994-1997. He was the co-recipient of the IEEE Acoustics, Speech, and Signal Processing Society Senior Paper Award in 1986 and the IEEE Signal Processing Society Paper Award in 1993. 